\title{
Auswirkungen einer frühen Förderung des Schriftspracherwerbs auf die schulischen Lese- und Rechtschreibleistungen
}

\section{Susanne Geyer}

Im vorliegenden Beitrag wird von einer Studie zur alltagsintegrierten Förderung der Schriftsprache am Übergang vom Kindergarten in die Grundschule berichtet. Überprüft wird, inwieweit es Effekte der Förderung im Kindergarten gibt, die sich auch noch am Ende des ersten Schulbesuchsjahres nachweisen lassen.

\section{Stand der Forschung - Fragestellung}

Bereits vor der Schule machen Kinder vielfältige, aber auch sehr unterschiedliche Lernerfahrungen über die Funktion und den Sinn von Lesen und Schreiben, die sie später beim systematischen Schriftspracherwerb miteinbringen. Vor allem die phonologische Bewusstheit und das Wissen über Schrift stehen in Zusammenhang mit dem schulischen Erfolg im Lesen und Rechtschreiben (Schneider 2008). Für das Lernen in der Schule ergibt sich demzufolge die Notwendigkeit, das Vorwissen der Kinder auszumachen, um entwicklungs- und bildungsförderliche Hilfen so gestalten zu können, dass die individuelle Schreib- und Leseentwicklung eines Heranwachsenden nicht aus dem Auge verloren wird. Es gibt verschiedene Ansätze dazu, wie die schriftsprachliche Entwicklung von Kindergartenkindern positiv beeinflusst werden kann: Strukturierte Trainingsprogramme richten sich als kompensatorische Fördermaßnahme gezielt und systematisch über einen begrenzten Zeitraum hinweg selektiv an benachteiligte Vorschulkinder mit ungünstigen Lernvoraussetzungen für den Erwerb von Lesen und Schreiben. Zwar lassen sich nachhaltige Effekte eines kombinierten Trainings der phonologischen Bewusstheit mit Buchstaben-Laut-Verbindungen nachweisen (Fischer / Pfost 2015), jedoch sind solche zusätzlichen Angebote nur bedingt mit der Vorstellung einer alltagsintegrierten Sprachbildung im Kindergarten zu vereinbaren. Umgekehrt deuten Studien aus dem angloamerikanischen Raum darauf hin, dass schriftsprachlich angereicherte Rollenspielecken (engl. Literacy-Center) und adaptive Entwicklungsangebote, wie das einer Anlauttabelle, Möglichkeiten bieten, auf die sprachliche Heterogenität im Alltag angemessen zu reagieren (Christie / Roskos 2006; Bodrova / Leong 2007). Befunde aus dem letzten Kindergartenjahr des Datensatzes, auf dem diese Teilstudie beruht, zeigen, dass alle Kinder am Schulanfang, unmittelbar nach Interventionsende, gleichermaßen im Bereich der phonologischen Bewusstheit im engeren Sinn von einer ,gut gemachten“ alltagsintegrierten Literacy-Förderung profitieren. Für das Arbeiten mit einer Anlauttabelle im Kindergarten ließ sich kein zusätzlicher Gewinn feststellen (vgl. Geyer et al. 2015). 
Der Schwerpunkt meines Beitrags liegt nun auf der Frage, ob sich ein vorschulisches Bildungsangebot, bei dem Kindergartenkinder in thematisch gestalteten Rollenspielecken zu Lese- und Schreibaktivitäten herausgefordert werden, auf die Lese- und Rechtschreibleistungen am Ende der ersten Jahrgangsstufe auswirkt und inwieweit die Anlauttabelle als zusätzliches Angebot v.a. für diejenigen Schüler hilfreich ist, die bereits im Kindergarten mehr Vorwissen besitzen.

\section{Methodik}

\subsection{Design}

Die vorliegende Studie basiert auf einem quasi-experimentellen Prä-Post-Kontrollgruppendesign. Als theoretische Grundlage für die Entwicklung der beiden alltagsintegrierten Treatments im letzten Kindergartenjahr diente das Konzept des „Literacy-Center“ (Kammermeyer / Molitor 2005). In thematischen Rollenspielecken wurden Spiele zur phonologischen Bewusstheit sowie Schrifterfahrungen initiiert. Zusätzlich wurde im zweiten Treatment eine Anlauttabelle angeboten. Eine Vergleichsgruppe absolvierte vor der Einschulung das Trainingsprogramm „Hören, lauschen, lernen 1“ (Küspert / Schneider 2008).

\subsection{Stichprobe}

Von den ursprünglich beteiligten 229 Vorschulkindern liegen Daten von 195 Kindern vor. Der Drop-out war hauptsächlich durch Wegzug, Zurückstellungen oder fehlende Elterneinwilligungen für die schulische Erhebung verursacht. Um einen vollständigen Datensatz zu erhalten, wurden die fehlenden Werte mithilfe eines modellbasierten Maximum-Likelihood-Verfahrens in einem Schritt mit dem EMAlgorithmus geschätzt und gleichzeitig imputiert (vgl. dazu Lüdtke et al. 2007). Dieses Verfahren bot sich an, da weniger als fünfzehn Prozent der Fälle fehlten (Wirtz 2004). Auswertbar sind die Daten aus 26 Klassen. Davon wurden 97 Kinder im letzten Jahr vor der Einschulung alltagsintegriert in einem Literacy-Center gefördert, 88 Kindern wurde zusätzlich eine Anlauttabelle angeboten und 44 Kinder nahmen am hochstrukturierten Trainingsprogramm „Hören, lauschen, lernen 1“ teil.

Im Zuge der Prüfung von Vortestunterschieden ergaben sich mit Ausnahme der Familiensprache keine signifikanten Differenzen zwischen den drei Untersuchungsgruppen, weder im Vorwissen (phonologische Bewusstheit eng, Buchstabenkenntnis/erstes Lesen) noch in den Hintergrundvariablen (Alter, Geschlecht, Intelligenz und Wortschatz). Zum Zeitpunkt der Follow-up-Testung waren die 
Kinder durchschnittlich 7,3 Jahre (SD = 0,3 Jahre) alt. Bezüglich des Sprachhintergrundes wurden 27 Kinder mit einer anderen Familiensprache als deutsch (ohne die bilingualen Kinder) klassifiziert. Der Post-hoc-Test nach Bonferroni bestätigt, dass Kinder mit einer nicht-deutschen Familiensprache häufiger in der Kontrollgruppe vertreten sind als im Treatment 1 ohne Anlauttabelle $(p=.041)$. In die weiteren Analysen wurde daher die Familiensprache als Kontrollvariable aufgenommen.

\subsection{Erhebungsinstrumente}

Um die schriftsprachlichen Vorkenntnisse zu Beginn des letzten Kindergartenjahres zu überprüfen, wurden Aufgaben zur phonologischen Bewusstheit im engeren Sinn (41 Items; Cronbachs $\alpha=.93$ ) und zu Buchstabenkenntnis/erstes Lesen (66 Items; Cronbachs $\alpha=.97$ ) aus dem Testverfahren ,wortgewandt und zahlenstark“ (Moser / Berweger 2007) eingesetzt. Im Nachtest wurde im Rechtschreiben die Potsdamer Bilderliste aus der frei zugänglichen Lernstandsanalyse ILeA (LISUM 2010) verwendet. Gewertet wurde, wie viele der 24 Wörter lauttreu richtig verschriftet wurden. Im Lesen kam am Ende der ersten Klasse die „Würzburger LeiseLeseprobe“ (Schneider et al. 2011) zum Einsatz, bei der die Schüler in einer vorgegebenen Zeit zu einem Wort das passende Bild herausfinden müssen. Die methodisch-didaktische Umsetzung des Erstlese- bzw. -schreibunterrichts in den Klassen wurde schriftlich über Selbsteinschätzungen der Lehrer dokumentiert um zu überprüfen, dass die Bedeutung des Schriftspracherwerbs in den Klassen vergleichbar war.

\subsection{Berechnungsmethoden}

Da im Kindergarten andere Aufgaben gestellt wurden als in der Schule, verbietet sich die Verwendung von Messwiederholungsmodellen. Aus diesem Grund werden zur Beantwortung der Frage nach dem Lerngewinn am Ende der ersten Jahrgangsstufe hierarchische Regressionsmodelle gerechnet, mit der zu Beginn des letzten Kindergartenjahres erhobenen phonologischen Bewusstheit als Kovariate auf Level 1. Um gleichzeitig auch noch Unterschiede zwischen Schulklassen auf Level 2 als Erklärungsvariablen (z.B. Erfahrung der Lehrkraft) auszuschließen, wird die Zugehörigkeit zu einer Schulklasse $(n=26)$ als Clustervariable modelliert. Die zentrale unabhängige Variable ist die Zugehörigkeit zu einem der drei vorschulischen Treatments. Für die Beantwortung der Frage nach dem Angebot einer Anlauttabelle für Kinder mit unterschiedlich großem Vorwissen, wird in einem Random-Intercept-and-Slope-Modell berechnet, ob sich die Stärke des Zusammenhangs zwischen Vor- und Nachtest in den drei Untersuchungsgruppen unterscheidet. 


\section{Ergebnisse}

Vergleicht man am Ende des ersten Schulbesuchsjahres auf Basis der vorschulischen Untersuchungsgruppen die Mittelwerte der im Kindergarten unterschiedlich geförderten Kinder (Tabelle 1), zeigen die Ergebnisse der hierarchischen Regressionsanalysen für das lauttreue Verschriften höhere Werte in den beiden LiteracyTreatmentgruppen als bei „Hören, lauschen, lernen 1“ $(p=.018)$. Dies gilt unter Kontrolle des Vorwissens und der Familiensprache. Die Effekte sind mit einem standardisierten Beta-Gewicht von .565 als moderat einzuschätzen. Post-hoc-Analysen deuten darauf hin, dass das zusätzliche Angebot einer Anlauttabelle einen positiven Effekt hat (mit einem standardisierten Betakoeffizienten von .627; $p=$ $.021)$ gegenüber der Förderung im Literacy-Center ohne Anlauttabelle $(\beta=.516$; $p=.017)$.

Tabelle 1. Lernergebnisse am Ende der ersten Jahrgangsstufe

\begin{tabular}{lcccccc}
\hline & $\begin{array}{c}\text { Literacy-Treat- } \\
\text { ment }\end{array}$ & \multicolumn{2}{c}{$\begin{array}{c}\text { Literacy-Treat }+ \\
\text { Anlauttabelle }\end{array}$} & $\begin{array}{c}\text { Würzburger Trai- } \\
\text { ning }\end{array}$ \\
\hline & $M$ & $S D$ & $M$ & $S D$ & $M$ & $S D$ \\
\hline Rechtschreiben* & 0.29 & 0.10 & 0.29 & 0.12 & 0.26 & 0.13 \\
\hline Lesefertigkeit & 0.84 & 0.12 & 0.83 & 0.16 & 0.77 & 0.19 \\
\hline & \multicolumn{1}{c}{$(\mathrm{n}=97)$} & & $(\mathrm{n}=88)$ & \multicolumn{2}{c}{$(\mathrm{n}=44)$}
\end{tabular}

Anmerkungen: $* p<.05 ; M$ : Mittelwert; $S D$ : Standardabweichung; $n=$ Anzahl der Erstklässler

In der Entwicklung der Lesefertigkeit lässt sich am Ende der ersten Jahrgangsstufe kein statistisch bedeutsamer Einfluss des vorschulischen Treatments mehr nachweisen. Mit standardisierten Betakoeffizienten von .196 (im Literacy-Treatment) bis .251 (im Literacy-Treatment mit Anlauttabelle) zeigen sich zwar positive Tendenzen auf den Lerngewinn im Lesen, die Unterschiede werden allerdings nicht überzufällig.

Erwartungsgemäß sagen die Werte in der phonologischen Bewusstheit im engeren Sinn (mit einem standardisierten $\beta$ von .416) zu Beginn des letzten Kindergartenjahres recht hoch die Fähigkeit voraus, am Ende der ersten Klasse lautgetreu zu verschriften. Vergleicht man den Zusammenhang zwischen dem Vorwissen (gemessen an der phonologische Bewusstheit eng) in den drei Gruppen so zeigt sich, dass er mit .479 am stärksten in der Würzburger Trainingsgruppe ist, gefolgt von 
$.394 \mathrm{im}$ Literacy-Treatment und am geringsten mit .377 im Literacy-Treatment mit Anlauttabelle. Diese Unterschiede sind jedoch nicht überzufällig.

\section{Diskussion}

Inhaltlich sprechen die Befunde eher für als gegen eine alltagsintegrierte Förderung der Schriftsprache vor der Schule - insbesondere, wenn man die Ergebnisse zum Ende des Kindergartens mitberücksichtigt. Dass im Unterschied zum lautgetreuen Schreiben kein Transfereffekt für das Lesen nachgewiesen werden konnte, könnte dadurch erklärt werden, dass beim Lesen das lexikalische Vorwissen von größerer Bedeutung ist, welches durch die alltagsintegrierte Förderung weniger explizit gefördert wurde. Leichte Vorteile der Arbeit mit einer Anlauttabelle ließen sich in unserer Studie erst am Ende des ersten Schulbesuchsjahres zeigen, was dafür sprechen könnte, dass der Anlauttabellen-Effekt in Alltagsförderkonzepten einige Zeit benötigt, um sichtbar zu werden. Dass vom Angebot einer Anlauttabelle auch längerfristig gesehen nicht nur ,die Guten“ profitieren, scheint zumindest mit Blick auf das Vorwissen ein Hinweis für das Gelingen einer individuellen Schreibentwicklung am Übergang vom Kindergarten in die Grundschule zu sein. Einschränkend muss allerdings angezeigt werden, dass nur wenig Informationen zur didaktischen Umsetzung des Lese- und Schreibunterrichts vorliegen, außer, dass er sich am gültigen Lehrplan orientierte. Von daher bleibt die Frage offen, inwiefern die „Passung“"zwischen einer schriftsprachlichen Förderung im Kindergarten und dem durchgeführten Anfangsunterricht der Lehrer wichtig ist.

Literatur:

Blömer, Daniel / Lichtblau, Michael / Jüttner, Ann-Kathrin / Koch, Katja / Krüger, Michaela / Werning, Rolf (Hrsg.) (2015): Perspektiven auf inklusive Bildung. Gemeinsam anders lehren und lernen. Wiesbaden: Springer

Bodrova, Elena, Leong, Deborah J. (2007): Tools of the Mind. The Vygotskian Approach to Early Childhood Education. Upper Saddle River: Pearson

Fischer, Melanie / Pfost, Maximilian (2015): Wie effektiv sind Maßnahmen zur Förderung der phonologischen Bewusstheit? Eine meta-analytische Untersuchung der Auswirkungen deutschsprachiger Trainingsprogramme auf den Schriftspracherwerb. In: Zeitschrift für Entwicklungspsychologie und Pädagogische Psychologie 47 (1). 35-51

Geyer, Susanne /Hartinger, Andreas / Kammermeyer, Gisela (2015): Alltagsintegrierte Förderung der Schriftsprache im Vorschulalter. In: Blömer et al. (2015): 243-248

Kammermeyer, Gisela / Molitor, Miriam (2005): Literacy Center - ein Konzept zur frühen Lese- und Schreibförderung in Theorie und Praxis. In: Roux (2005): 130-142 
Küspert, Petra / Schneider, Wolfgang (2008): Hören, lauschen, lernen 1. Sprachspiele für Kinder im Vorschulalter. Würzburger Trainingsprogramm zur Vorbereitung auf den Erwerb der Schriftsprache. Göttingen: Vandenhoeck \& Ruprecht

LISUM (Landesinstitut für Schule und Medien Berlin-Brandenburg) (2010): Individuelle Lernstandsanalysen. Schülerheft Deutsch 2. http://bildungsserver.berlin-brandenburg.de/fileadmin/bbb/unterricht/lernbegleitende_Diagnostik/ilea/2010/2SchuelerDeutsch.pdf 2010 (Abruf vom 04.03.2016)

Lüdtke, Oliver / Robitzsch, Alexander / Trautwein, Ulrich / Köller, Olaf(2007): Umgang mit fehlenden Werten in der psychologischen Forschung. In: Psychologische Rundschau 58 (2). 103-117

Moser, Urs / Berweger, Simone (2007): wortgewandt \& zahlenstark. Test zur Ermittlung des Lern- und Entwicklungsstands bei 4- bis 6-Jährigen. Zürich: Kantonaler Lehrmittelverlag

Roux, Susanna (Hrsg.) (2005): Pisa und die Folgen. Sprache und Sprachförderung im Kindergarten. Landau: Empirische Pädagogik

Schneider, Wolfgang (Hrsg.) (2008): Entwicklung von der Kindheit bis zum Erwachsenenalter. Befunde aus der Münchner Längsschnittstudie LOGIK. Weinheim: Beltz

Schneider, Wolfgang / Blanke, Iris / Faust, Verena / Küspert, Petra (2011): Würzburger Leise-Leseprobe Revision. Ein Gruppentest für die Grundschule. Göttingen: Hogrefe

Wirtz, Markus (2004): Über das Problem fehlender Werte. Wie der Einfluss fehlender Informationen auf Analyseergebnisse entdeckt und reduziert werden kann. In: Rehabilitation 43. 109-115 